\title{
Inside-Out Rotary Ramjet Turbogenerator
}

\author{
Werner J.A. Dahm ${ }^{1}$, Andrew P. Lapsa ${ }^{2}$ and Peter E. Hamlington ${ }^{2}$ \\ Laboratory for Turbulence \& Combustion (LTC), Department of Aerospace Engineering, \\ The University of Michigan, Ann Arbor, MI 48109-2140 USA
}

\begin{abstract}
A method for implementing the Brayton cycle to provide a practical rotary ramjet engine/generator based on a fundamentally "inside-out" approach is presented. Such a rotary ramjet turbogenerator eliminates the need for the conventional bladed compressor and turbine used in traditional gas turbine engines. It replaces these with a single inside-out supersonic circumferential rotor having integrated varying-area shaped ceramic ramjet channels in its radially inward surface, in which compression, combustion and expansion occur. The inside-out design places all rotating parts under compressive centrifugal loading, allowing ceramic fabrication of the high-temperature ramjet channel sections to enable operation at increased combustor exit temperatures with substantially reduced cooling requirements. The increased combustor temperature leads to increased maximum power and increased thermal efficiency when operating at maximum power. Furthermore, in this inside-out approach the centripetal acceleration acting on the flow in the ramjet channels causes high-density reactants to be forced into the radially inward-facing combustor, and low-density combustion products to be forced out of the combustor. This greatly assists in mixing and flameholding without the need for vortex generators or similar devices. The advantages of the inside-out approach over previous rotary ramjet systems become increasingly important as the rotor radius is decreased, since the centripetal acceleration then becomes increasingly large.
\end{abstract}

\section{Introduction}

$\mathrm{C}$ ONVENTIONAL gas turbine engine systems for turbogenerator, shaft power, and aeropropulsion applications typically require a compressor, driven by a turbine, to achieve the high compression ratios needed for acceptable operating efficiencies and power output. Most of the mass in such systems comes from the compressor and turbine, which in turn limits the mass-specific power achievable by traditional gas turbine-based systems. The requirements for a compressor and turbine also introduce limits on the performance of such conventional systems. In particular, material temperature limitations for the turbine blades restrict the maximum permissible turbine inlet temperature, which requires operation at large excess air levels and thereby limits the efficiency and power achievable with such conventional systems. Typically, air must be bled from the compressor to flow through intricate internal cooling channels within the leading rows of turbine blades, and then through fine holes in the blade surfaces to provide film cooling to maintain blade temperatures within acceptable limits. The bleed air used for turbine blade cooling further detracts from the overall efficiency of the system, and blade cooling losses can be a source of turbine failures. The need for a traditional bladed compressor and turbine thus contributes substantially to the cost of maintaining such systems, and often ultimately limits the usable life of such systems. Moreover, the complex blade shapes needed for high-efficiency bladed compressors and turbines, and the cooling channels and film cooling holes within these turbine blades, contribute significantly to the overall complexity and cost of developing and fabricating Brayton-cycle systems based on the conventional gas turbine approach.

By contrast, in a ramjet system the need for such a bladed compressor is removed by the high ram pressures that can be achieved in forward flight when the flight Mach number is sufficiently large, typically above about $M=1.4$. Removing the compressor further eliminates the need for the turbine as well, with a result being that the mass of a ramjet engine is far lower than that of a conventional gas turbine producing the same thrust. Eliminating the rotating

\footnotetext{
${ }^{1}$ Professor of Aerospace Engineering; Head, Laboratory for Turbulence \& Combustion (LTC); AIAA Fellow.

${ }^{2}$ Graduate Student Research Assistant, Department of Aerospace Engineering; AIAA Student Member.
} 
bladed turbine also allows the combustor in a ramjet system to be operated at higher overall combustion temperatures, and thereby provides higher efficiencies than conventional bladed turbine engines can achieve at the same compression ratio. Moreover, without the need for a compressor or turbine the costs of developing, producing and maintaining a ramjet system can be far lower that for traditional gas turbine engines. However operation of a ramjet engine requires that the Mach number of the approaching flow must first be brought to a sufficiently high value, typically by increasing the forward flight speed of the vehicle by some other propulsion system, to provide enough ram compression for the ramjet to produce net positive thrust. For this reason, ramjets to date have been largely restricted to specialized aeropropulsion applications, most notably in tactical missiles and in certain highspeed air vehicles.

Due to the many advantages of ramjet-based systems, numerous approaches have long been considered to allow ramjets to be used in a wider range of applications, including stationary systems or systems that can start from rest. Many of the earliest attempts were based on rotating approaches, often with conventional ramjet engines positioned on rotatable arms so that the ramjet thrust would provide the torque to drive the rotation, while the rotating motion would provide the ram compression needed for the ramjets to operate. However to date no such systems have seen significant use in practice, in many cases for simple mechanical reasons.

A somewhat different and more promising approach $[1,2]$ has recently sought to integrate ramjet flowpaths in the outer rim of a high-speed rotating disk to produce a ramjet-based power generator. In that case, two-dimensional ramjet flow channels were formed in the exterior or radially outer-most surface of the rotor disk and were closed by the stationary interior surface of the engine case. The ramjet flowpaths were oriented in the rotor rim at a helix angle that allowed a low-speed axial flow along the direction of the axis of rotation of the high-speed rotor. The rotating motion of the disk thus provided a continuous flow of premixed fuel-air mixture into the ramjet flowpath inlets. Since the disk rotated at a rim speed near $M=2$, the helical ramjet flowpaths provided sufficient ram compression to produce net positive thrust along the helix angle from combustion of the reactant mixture. Due to the high combustion temperatures and the high tensile stresses produced by the rotating motion of the ramjet channels in the rotor rim, conventional nickel-based alloy materials were used in the combustor and expansion sections of the ramjet flowpaths in conjunction with traditional air film cooling. Tests with such a device having a 6-ft diameter rotor showed that vortex generators and bluff body flameholders were needed to augment mixing and maintain combustion in the rotating combustors. While those tests did not produce net output power [1,2], they showed that in principle such a rotating ramjet concept is feasible.

Here we describe an improved approach for developing a practical rotary ramjet system for aeropropulsion and power generation applications. The present approach is related to that in [1,2], but differs in several very important ways. It seeks to eliminate the bladed compressor and turbine in a Brayton-cycle turbogenerator, and thereby to remove much of the weight, cost, complexity, and endurance limitations of conventional gas turbine-based systems. In the present approach these components are replaced with an "inside-out" ceramic ramjet rotor that operates at sufficiently high supersonic speeds to allow efficient compression-combustion-expansion within varying-area ramjet flowpaths formed in the ceramic rotor. Unlike the rotor in [1,2], the present rotor is based on an "inside-out" design, shown in Figs. 1-5, in which it rotates around a simple concentric ceramic stator. The inside-out design has the advantage of placing the ramjet flowpath material on the inside rim of the rotor under purely compressive centrifugal loading. This in turn allows high-temperature ceramic materials - which typically have much higher compressive strength than tensile strength - to be used through the ramjet channels. Such an all-ceramic flowpath allows for substantially reduced cooling requirements in the combustor and expansion sections, and may even entirely eliminate the need for internal cooling channels or film cooling holes in such systems. The "inside-out" approach thus greatly simplifies design and fabrication of practical rotary ramjet systems. Moreover, the all-ceramic ramjet flowpath made possible by the purely compressive loading in the present inside-out approach allows substantially higher combustor exit temperatures than are possible with either a conventional bladed turbine or with the metal-based "outside-out" ramjet rotor in $[1,2]$. The increased combustor temperature provides a substantial increase in maximum power and increased thermal efficiency when operating at maximum power. Moreover, in addition to the present "inside-out" rotor being made of high-temperature ceramic material, the fact that it is subjected to compressive loading allows further increases in the combustor exit temperature, to achieve higher power and efficiencies than would otherwise be possible.

Equally important, in the present "inside-out" rotary ramjet approach the centrifugal forces acting on reactants and products as they flow through the rotating ramjet channels are precisely opposite to those in [1,2]. In the insideout approach the centripetal acceleration causes high-density reactants to be naturally forced into the radially inward-facing combustor, which greatly assists with mixing and flameholding without the need for vortex generators 


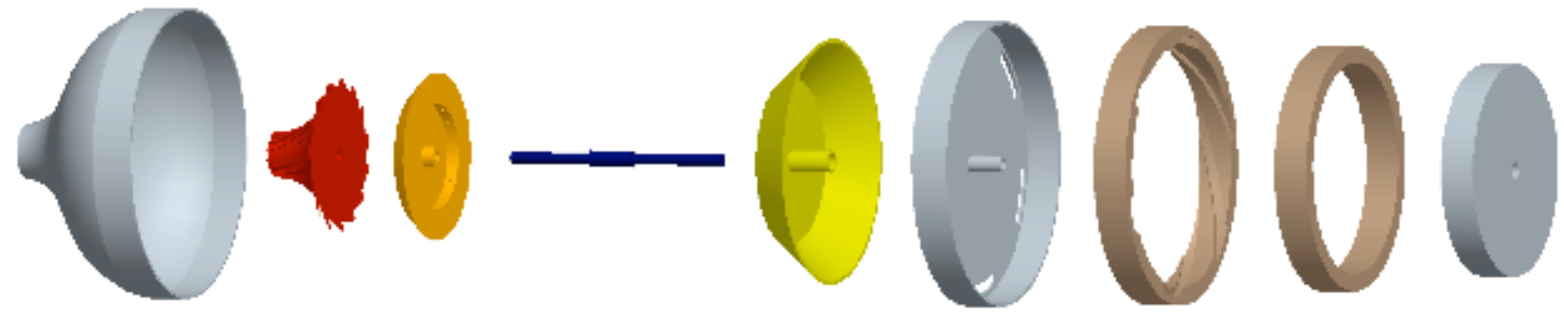

Figure 1. Schematic showing major components of inside-out rotary ramjet approach, including (from left to right) housing, impeller, fuel slinger, shaft, spacer, metal support rim, ceramic rotor, ceramic stator, and stator frame.

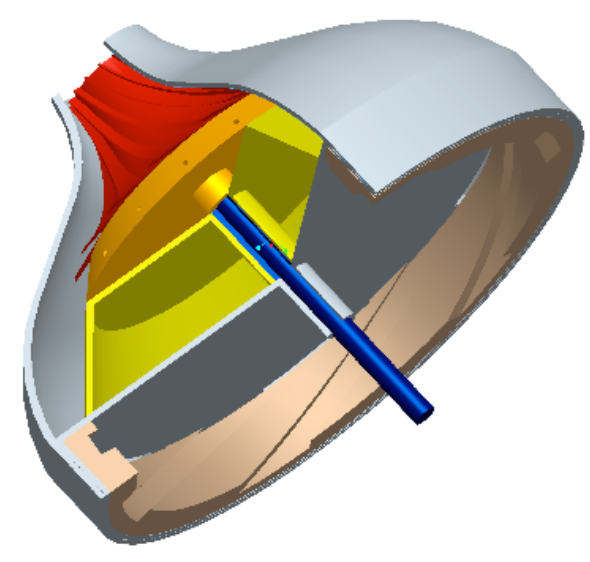

Figure 2. Assembly view of major components, showing all but last two components in Fig. 1.

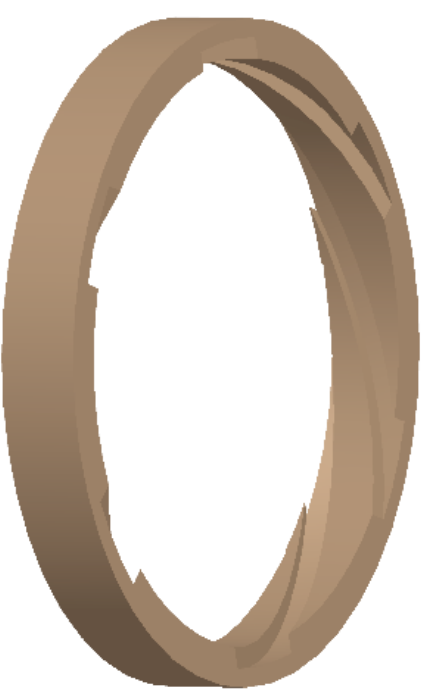

Figure 4. Schematic of ceramic rotor, showing inside-out helical ramjet channels.

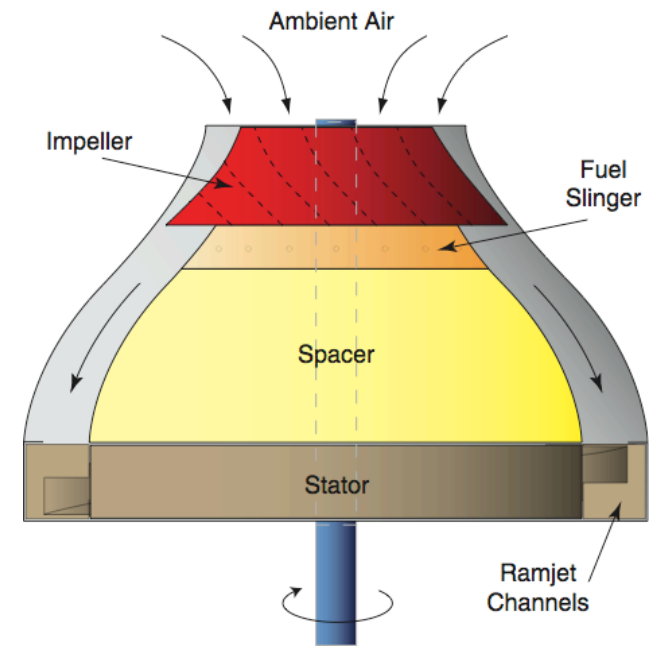

Figure 3. Assembly schematic showing creation of fuel-air mixture and flow into ramjet channels.

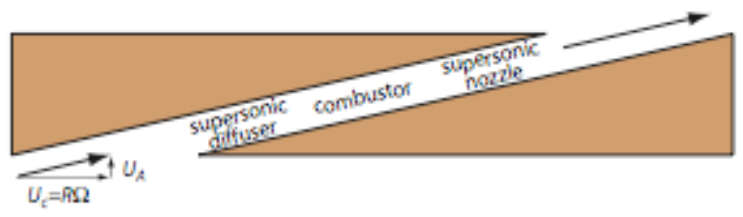

(a)

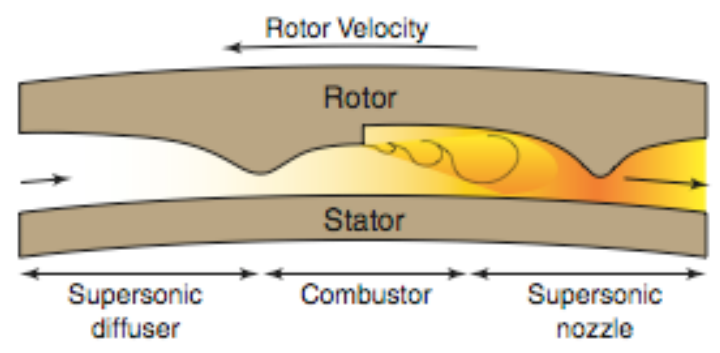

(b)

Figure 5. Schematic of ramjet flowpath in helical channels (top) and cross-sectional view (bottom). 
or similar devices. By contrast, in the "outside-out" approach used in $[1,2]$ the centrifugal forces acting on the flow through the ramjet channels due to the rotation inherently caused the radially outward-facing combustor to fill with low-density combustion products. This in turn required the use of vortex generators to assist in mixing fresh reactants into the bluff-body combustor. The present "inside-out" approach further simplifies the development and fabrication of practical rotary ramjet systems by reducing or eliminating the need for such vortex generators and other devices to assist with mixing and flameholding in the combustor. Moreover, it appears likely that the strong tendency of the combustor in the "outside-out" approach to fill with low-density combustion products under the large centripetal accelerations present in rotating ramjet systems may have been a key reason why the approach in $[1,2]$ did not produce net output power.

For these reasons, inside-out rotary ramjet turbogenerators of the type described herein can eliminate much of the weight, cost and complexity of conventional gas turbine-based systems, and can achieve higher power density, increased efficiency, increased durability and significantly reduced cost over previous rotary ramjet systems. These advantages of the inside-out approach over previous rotary ramjet systems become increasingly important as the rotary ramjet size is decreased, since maintaining the required rotor rim speed at sufficiently high Mach number requires increased rotation rates - and thus increased centripetal accelerations - as the rotor radius is decreased.

\section{The Inside-Out Rotary Ramjet Configuration}

This section summarizes the basic approach and principle of operation of this inside-out rotary ramjet engine. Figure 1 gives an exploded view of one practical arrangement for implementing this type of system, Fig. 2 shows an assembly view of the same arrangement, and Figs. 3-5 shows schematics of various key aspects of the inside-out geometry. Beginning at the front of the central shaft, a rotating impeller rotates with the shaft to induce a nominally axial air flow into the housing. A fuel slinger mounted behind the impeller also rotates with the shaft. Liquid fuel is supplied to the slinger face through a non-rotating spacer, which also provides the principal bearing support for the rotating shaft. The liquid fuel flows along the slinger face and issues in a thin film from small holes along its rim, where the high rate of rotation provides centrifugal atomization of the fuel into fine droplets. The fuel droplets issue into the nominally axial air flow induced by the impeller, where they rapidly vaporize and mix in the cavity between the spacer and the housing to provide an axial flow of a lean premixed prevaporized reactant stream.

The resulting reactant stream flows axially above the spacer toward a rotor assembly mounted on the same rotating shaft. The rotor assembly consists of a metal rim that provides structural support for an inside-out ceramic rotor. The ceramic rotor has shaped ramjet channels formed on its inside periphery; these channel run helically around the inside periphery of the rotor, as shown in Fig. 4. This ceramic rotor is subjected to purely compressive loading under the large centripetal acceleration it experiences at the design rotation rate. Inside this ceramic rotor assembly is a stator assembly, consisting of a ceramic stator formed around the periphery of a metal rim that supports the ceramic stator. The ceramic rotor and stator parts form face-seals where they meet, with the close fit of the rotating ceramic rotor around the ceramic stator effectively sealing the ramjet channels on the inside periphery of the rotor. The ramjet channels remain open at their exit where they intersect the downstream face of the ceramic rotor. They also remain open at their entrance where they intersect the upstream face of the ceramic rotor. The metal rim of the rotor contains small openings that are aligned with these entrances to the ramjet channels, thus permitting the nominally axial reactant flow above the spacer to enter these channels.

The entire rotor assembly, including the ramjet channels, rotates at the design shaft rotation rate. The high shaft rotation rate and the relatively large radial location of the channel entrances produce a supersonic azimuthal velocity of each channel entrance relative to the approaching reactant stream. The reactant stream thus flows into the channels at a relatively low axial velocity, but at an azimuthal velocity that corresponds to a sufficiently high inlet Mach number to provide for efficient ramjet propulsion within the ramjet channels. As shown in Fig. $5 a$, relative to the rotating ramjet channels the reactants are flowing into these channels at the design Mach number at an angle set by the ratio of the axial and azimuthal velocities. The channels are shaped to present a classical ramjet flowpath in the frame of the reactant stream flowing through them, as shown in Fig. $5 b$. The ramjet channels thus have varying cross-sectional area that forms a supersonic diffuser immediately past their entrance. The converging-diverging diffuser is shaped to provide near-isentropic compression of the reactant stream to the subsonic combustor Mach number at the end of the diffuser. This subsonic reactant stream then continues to flow into a combustor formed in the ceramic channel, where the premixed reactants burn rapidly under highly turbulent conditions. The combustor is shaped to provide effective stabilization of the reaction zone. The combustion product stream flows from the combustor into a converging-diverging nozzle that provides near-isentropic expansion of the products to ambient 
pressure at the exit of the ramjet channels. The combustion products issue from the exit of the ramjet channels at the back face of the ceramic rotor.

The difference in momentum flux of the reactant inflow into the entrance to the ramjet channels and the combustion product outflow from the exit of the channels induces a net thrust along the channels. This reaction force has a large component that acts along the azimuthal direction to produce a torque on the rotor that is transferred to the shaft. Part of this reaction torque provides the shaft power that drives the impeller and offsets the power dissipated in the bearings and by viscous stresses on the rotor surface to maintain the design shaft rotation rate. The remaining torque provides the net shaft power produced by the system; this shaft power may be used to drive a generator when the system is implemented in a turbogenerator, or to drive a fan or propeller when the system is implemented in a turbofan or turboprop. Alternatively, when implemented as a pure turbojet there is no requirement for excess shaft power, and thus the angle of the ramjet channels is made larger, with the resulting axial component of the reaction force providing a relatively large net axial thrust from the engine.

The implementation as a turbogenerator furthermore allows the electrical generator to be used as a motor during startup to drive the shaft to a sufficiently high initial rotation rate, corresponding to rotor rim Mach number of about $M_{0}=1.2$. At this initial rotation rate, the ramjet channels achieve sufficient compression to produce positive net thrust. Once the combustor achieves net thrust, the motor/generator is switched from motor to generator mode; at this point the reaction torque on the rotor provides further spin-up to the nominal operating rotation rate that corresponds to the design inlet Mach number $M_{0}$. The steady state operating point is reached at the rotation rate for which the resulting compression ratio at that rotor rim speed produces sufficient reaction torque on the rotor to offset the viscous drag and bearing losses, and provide net shaft power to the generator.

As the above description shows, this system is still based on the Brayton cycle, but differs from conventional gas turbine engines and from previous rotary ramjet approaches in several important ways. It eliminates the traditional bladed compressor and turbine that accounts for much of the weight, cost, complexity, and endurance limitations of conventional gas turbine engines, and replaces these with a far simpler ceramic ramjet rotor. This ceramic rotor has an "inside-out" design, in which it rotates around a very simple ceramic stator. This provides an all-ceramic flowpath that allows much higher combustion temperatures entering the expansion section than are possible with a bladed turbine or with conventional rotary ramjet engines.

\section{Brayton Cycle Analysis and Sensitivities}

In this section, we use a simple thermodynamic cycle analysis to obtain preliminary estimates of the performance of such a rotary ramjet system, and to identify the relative sensitivity of the performance to losses in the compression, combustion and expansion sections of the ramjet channels. In particular, we use classical results for a nonideal Brayton cycle to examine how the thermal efficiency and net output power per unit mass of reactant supplied to the ramjet channels depends on the rotor rim radius $R$ and rotation rate $\Omega$. These two parameters determine the rim speed $U_{C}=R \Omega$, and together with the sound speed $a_{0}=\left(\gamma R T_{0}\right)^{1 / 2}$ determine the Mach number $M_{0}$ at which the reactant mixture approaches the rotating ramjet inlets. Note that, as suggested in Fig. $5 a$, the nominal axial velocity $U_{A}<<U_{C}$, and thus we consider the inlet Mach number to be $M_{0}=U_{C} / a_{0}$.

Using subscript " 0 " to denote conditions approaching the ramjet inlets and subscript " 3 " for conditions exiting the combustor, then following $[3,4]$ the thermal efficiency can be expressed in terms of the inlet Mach number $M_{0}$ as

$$
\eta_{t h}=\eta_{b}\left\{1-\frac{T_{3}-\eta_{e} T_{3}\left(\frac{(\gamma-1) M_{0}^{2}}{\left.2+(\gamma-1) M_{0}^{2}\right)}\right)-T_{0}}{T_{3}-\frac{T_{0}}{\eta_{c}}\left(\frac{\gamma-1}{2} M_{0}^{2}+\eta_{c}\right)}\right\}
$$

where $T_{0}$ is the static temperature of the reactants approaching the ramjet channels, $T_{3}$ is the combustor exit temperature, and $\gamma$ is the specific heat ratio, here taken to be 1.3. The adiabatic efficiencies are denoted by $\eta_{c}$ for the compression section and $\eta_{e}$ for the expansion section, and the combustion efficiency is denoted by $\eta_{b}$. Similarly, the net work per unit mass of reactant mixture supplied to the ramjet inlets can be expressed as 


$$
\frac{w_{n e t}}{c_{p} T_{0}}=\frac{\eta_{t h}}{\eta_{b}}\left(\frac{T_{3}}{T_{0}}-\frac{1}{\eta_{c}} \frac{\gamma-1}{2} M_{0}^{2}-1\right),
$$

where $c_{p}$ denotes the specific heat of the gases flowing through the ramjet channels, and is here taken to be constant. The thermal efficiency and mass-specific work in (1) and (2) allow an initial parametric assessment of such a system, particular an assessment of the relative sensitivities of the system performance to compression efficiency, combustion efficiency, expansion efficiency, and combustor temperature.

Figure 6 shows the baseline performance of a system in which the flowpath section efficiencies $\eta_{c}, \eta_{b}$ and $\eta_{e}$ are all taken to be $90 \%$, and the combustor exit temperature $T_{3}$ is taken as $1750 \mathrm{~K}$. For both the thermal efficiency and the mass-specific net work, the results for the assumed baseline system are compared with corresponding results for an ideal system having the same $T_{3}$ but having all $\eta_{i}=1$ for the section efficiencies. The optimal rotation rate corresponding to maximum net output power is seen to correspond to an inlet Mach number $M_{0}$ slightly below 3 . Near the maximum power operating point, the performance of the nonideal system given by the solid lines is seen to be substantially below that of the dotted lines corresponding to an ideal system. The nonideal thermal efficiency near the maximum power operating condition is slightly below $40 \%$.

Figures 7-10 each examine the effect of varying one of the quantities $\eta_{c}, \eta_{b}, \eta_{e}$ and $T_{3}$ while holding all others constant at their baseline values. In Figs. 7-9 the ideal net work and ideal thermal efficiency corresponding to $\eta_{i}=1$ are also shown as dotted lines for comparison. In Fig. 10, these ideal values change as $T_{3}$ is varied, but for visual clarity only the baseline $\left(T_{3}=1750 \mathrm{~K}\right)$ ideal solution is shown. Note in Fig. 8 that the specific net work is not affected by the combustion efficiency $\eta_{b}$; this is due to the fact that the analysis is for a $T_{3}$-limited cycle, and thus irrespective of the completeness of combustion the combustor exit temperature remains constant at this $T_{3}$ value. Although the net work is not affected, the thermal efficiency is obviously reduced as $\eta_{b}$ decreases.

By comparing the results in Figs. 7-10, it can be seen that the thermal efficiency is most strongly affected by the expansion section efficiency $\eta_{e}$, followed in order of decreasing sensitivity by $\eta_{b}, \eta_{c}$ and $T_{3}$. Moreover it can also be seen that the mass-specific net work is affected in order of decreasing sensitivity by $T_{3}, \eta_{e}, \eta_{c}$, and $\eta_{b}$. While the effect of $T_{3}$ on $w_{\text {net }}$ is greatest, it is apparent in Fig. 10 that this is primarily a result of its effect on the ideal net work, since $T_{3}$ is seen to have very little influence on the differences between the ideal and nonideal overall thermal efficiencies $\eta_{t h}$ in Fig. $10 b$.

These results not only reaffirm the importance of maximizing the combustor exit temperature $T_{3}$ to maximize the power output of such a system, and also reveal two of the most important design advantages of the inside-out rotary ramjet approach. Owing to the inherently compressive centrifugal loading to which the ramjet flowpath material is subjected in an inside-out design, the entire flowpath can be made of ceramic materials. As a result, $T_{3}$ can be made substantially higher for the same degree of internal and film cooling than is possible in an outside-out design; in the latter, traditional lower-temperature metal alloys or other high tensile strength materials must be used due to the tensile stresses produced by centrifugal forces. The advantages of maximizing $T_{3}$ are apparent in Fig. 10a. Moreover, the ability to use ceramic material in the expansion section of the inside-out ramjet flowpath may lead to additional improvements in the expansion efficiency $\eta_{e}$, which is seen in Fig. $9 b$ to have the largest influence on the thermal efficiency $\eta_{t h}$. This is because the highest wall heat flux typically occurs in the throat of the expansion section, where temperatures and velocities are simultaneously high. That combination can lead to a gradual loss over time of throat wall material when operating near the maximum temperature limit of the material. The result is a gradual increase in the throat area $A^{*}$, which in turn leads to increasingly underexpanded flow at the end of the expansion section due to the change in nozzle exit area ratio $A_{e} / A^{*}$. The high-temperature capabilities of ceramic materials can avoid such throat degradation by allowing operation further below the maximum temperature limit of the wall material, even while allowing an increase in the combustor exit temperature $T_{3}$. Thus the ability to use high-temperature ceramic materials throughout the ramjet channel, including both the combustion and expansion sections, allows for both a direct increase in maximum power due to the $T_{3}$ effect on $w_{\text {net }}$, as well as a secondary increase in thermal efficiency via the $\eta_{e}$ effect on $\eta_{t h}$ as a result of maintaining the optimal $A_{e} / A^{*}$.

\section{Additional Considerations}

The inside-out approach described in $\S \S \mathrm{I}$ and II for developing a practical rotary ramjet engine is central to enabling an all-ceramic flowpath, which in turn enables the increases in maximum power and thermal efficiency noted in §III. Additionally, the ability to use high-temperature ceramic materials in the inside-out ramjet flow 

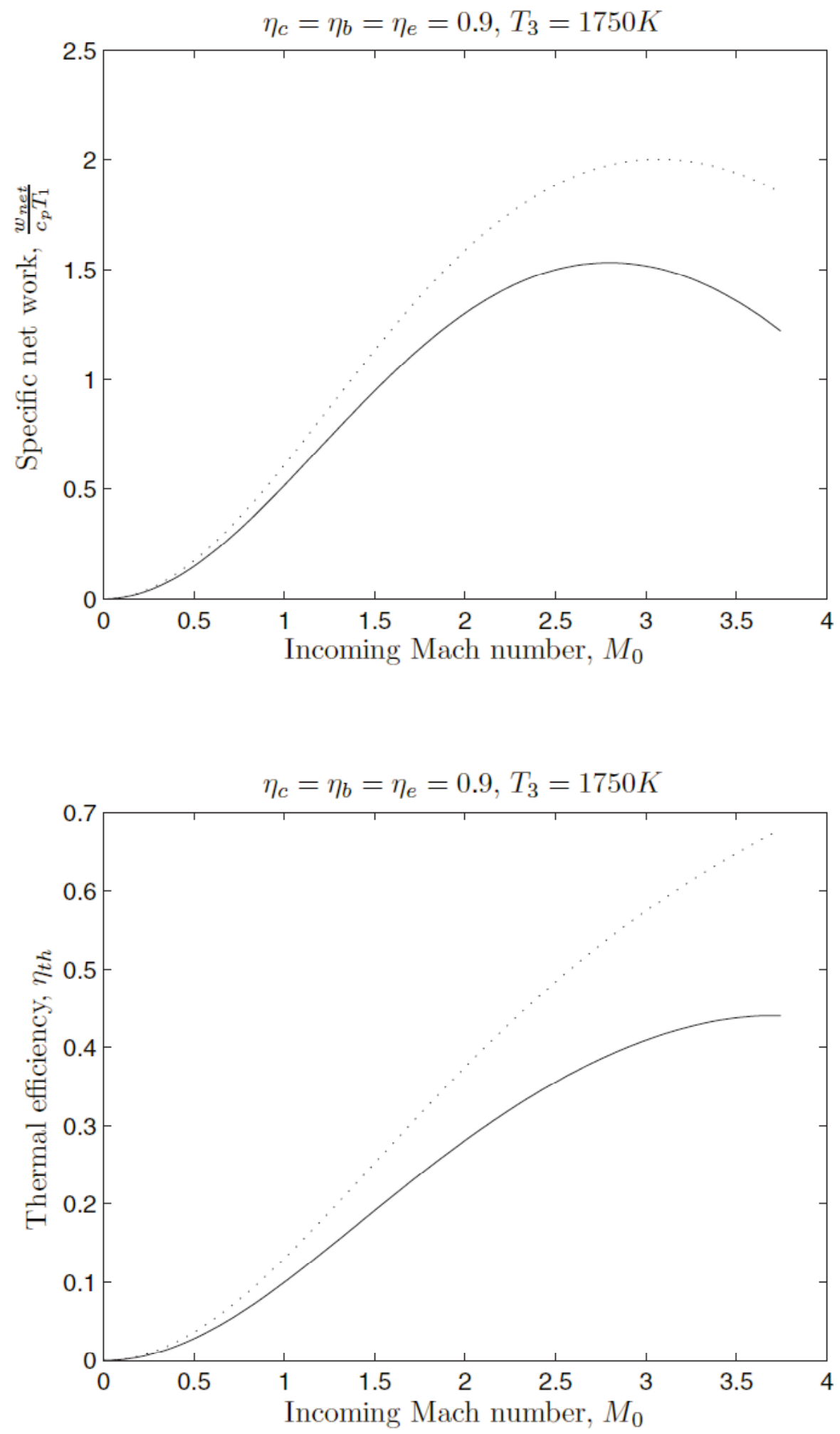

Figure 6. Net work per unit mass of reactants (top) and thermal efficiency (bottom) for ideal system (dotted) and for baseline non-ideal system with all efficiencies at $90 \%$ and $1750 \mathrm{~K}$ combustor exit temperature (solid). 

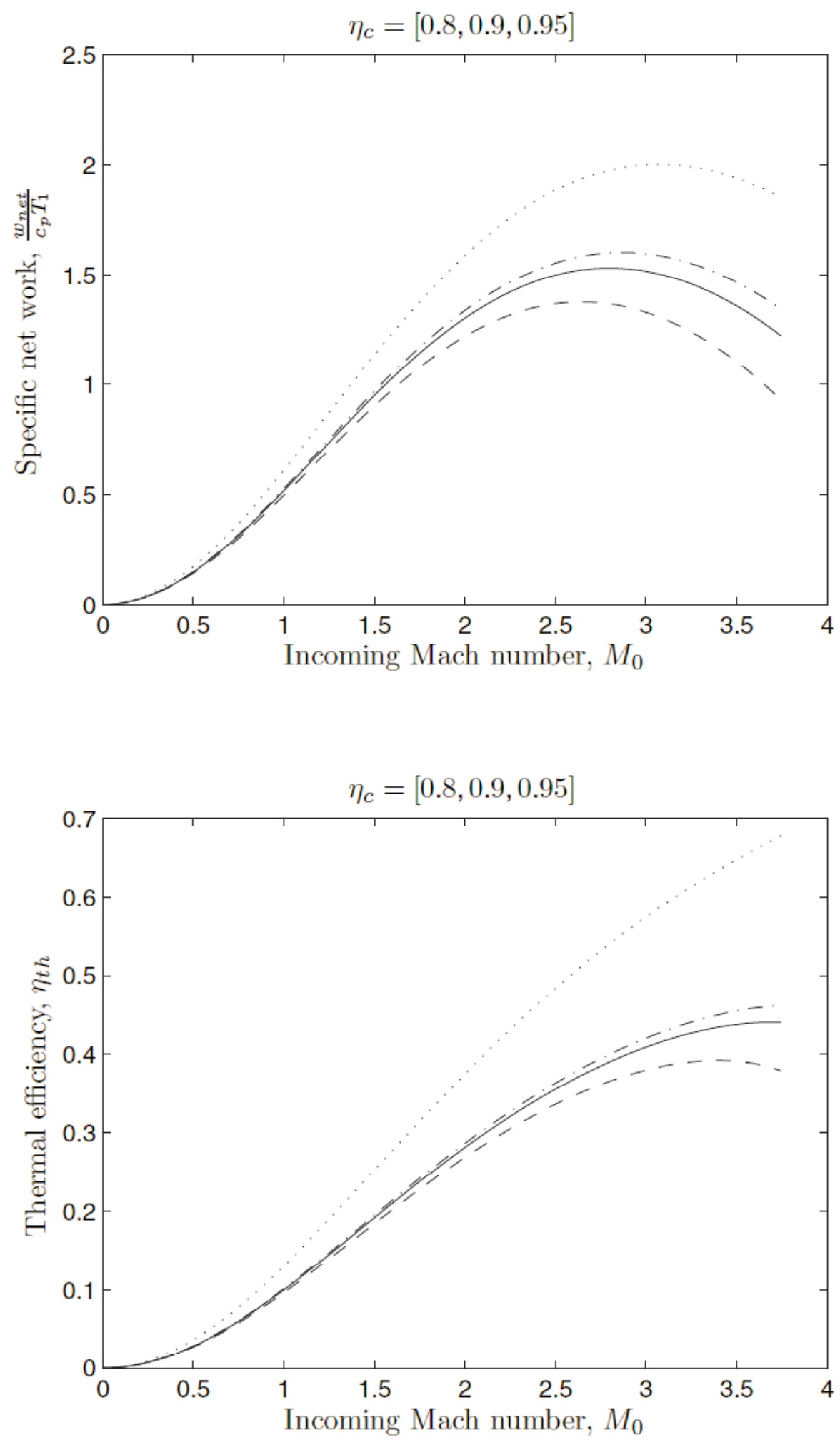

Figure 7. Effect of compression efficiency $\eta_{c}$ on net work per reactant mass (top) and thermal efficiency (bottom), showing ideal (dotted) and non-ideal system performance with $\eta_{c}=80,90$ and $95 \%$ (remaining lines) for baseline $1750 \mathrm{~K}$ combustor exit temperature and values $\eta_{b}=\eta_{e}=90 \%$. 

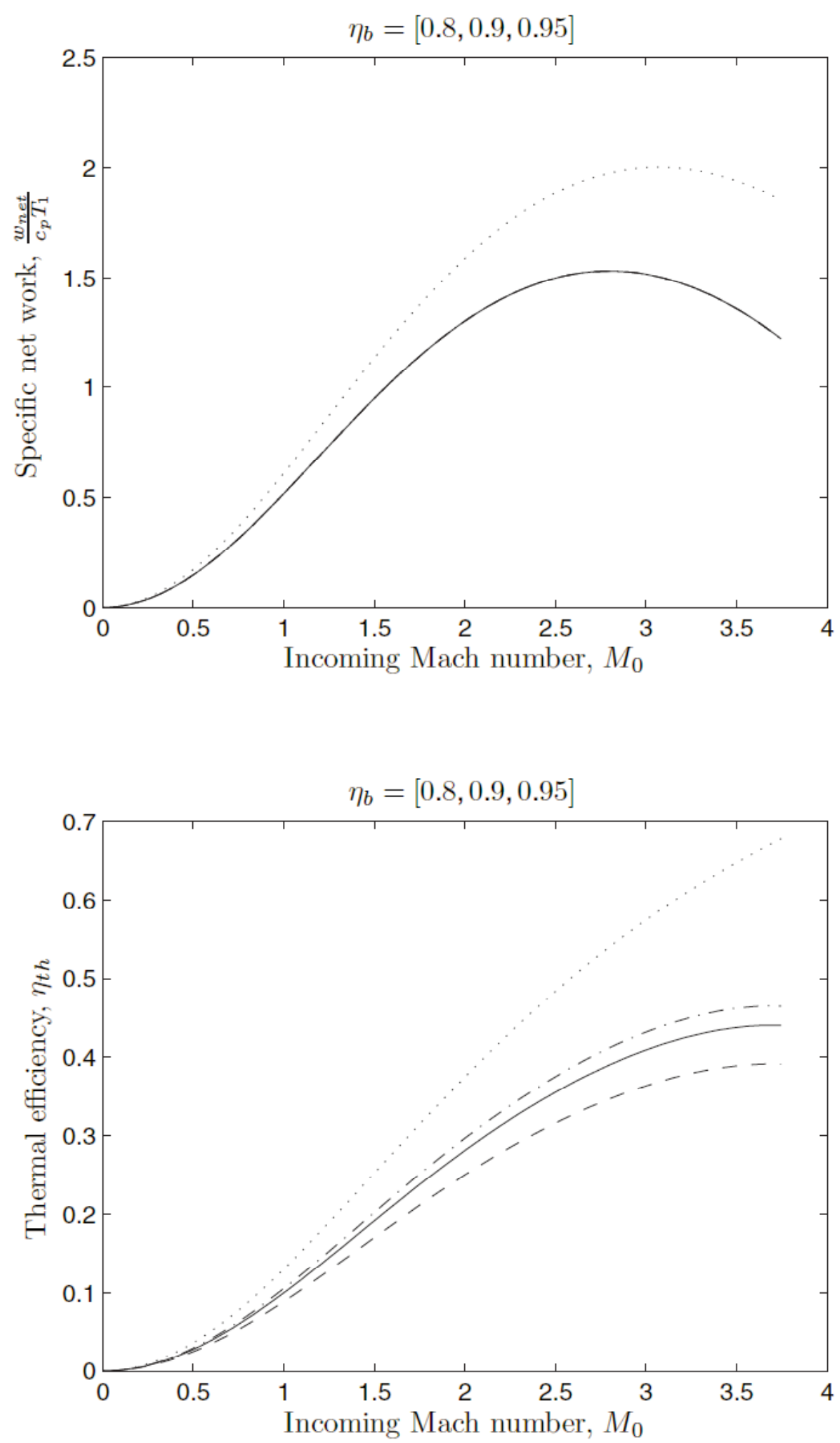

Figure 8. Effect of combustion efficiency $\eta_{b}$ on net work per reactant mass (top) and thermal efficiency (bottom), showing ideal (dotted) and non-ideal system performance with $\eta_{b}=80,90$ and $95 \%$ (remaining lines) for baseline $1750 \mathrm{~K}$ combustor exit temperature and values $\eta_{c}=\eta_{e}=90 \%$. 

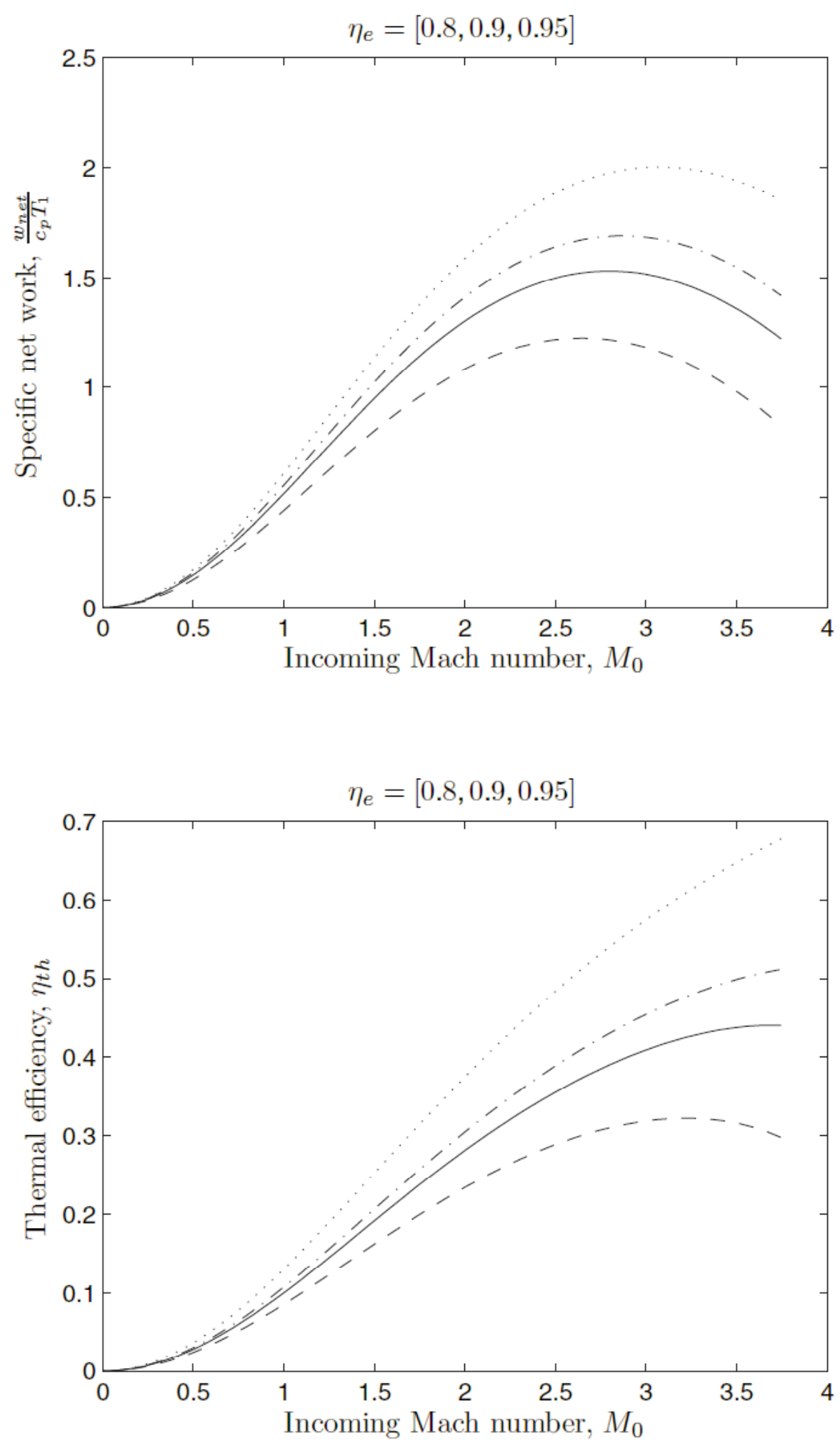

Figure 9. Effect of expansion efficiency $\eta_{e}$ on net work per reactant mass (top) and thermal efficiency (bottom), showing ideal (dotted) and non-ideal system performance with $\eta_{e}=80,90$ and $95 \%$ (remaining lines) for baseline $1750 \mathrm{~K}$ combustor exit temperature and values $\eta_{c}=\eta_{b}=90 \%$. 

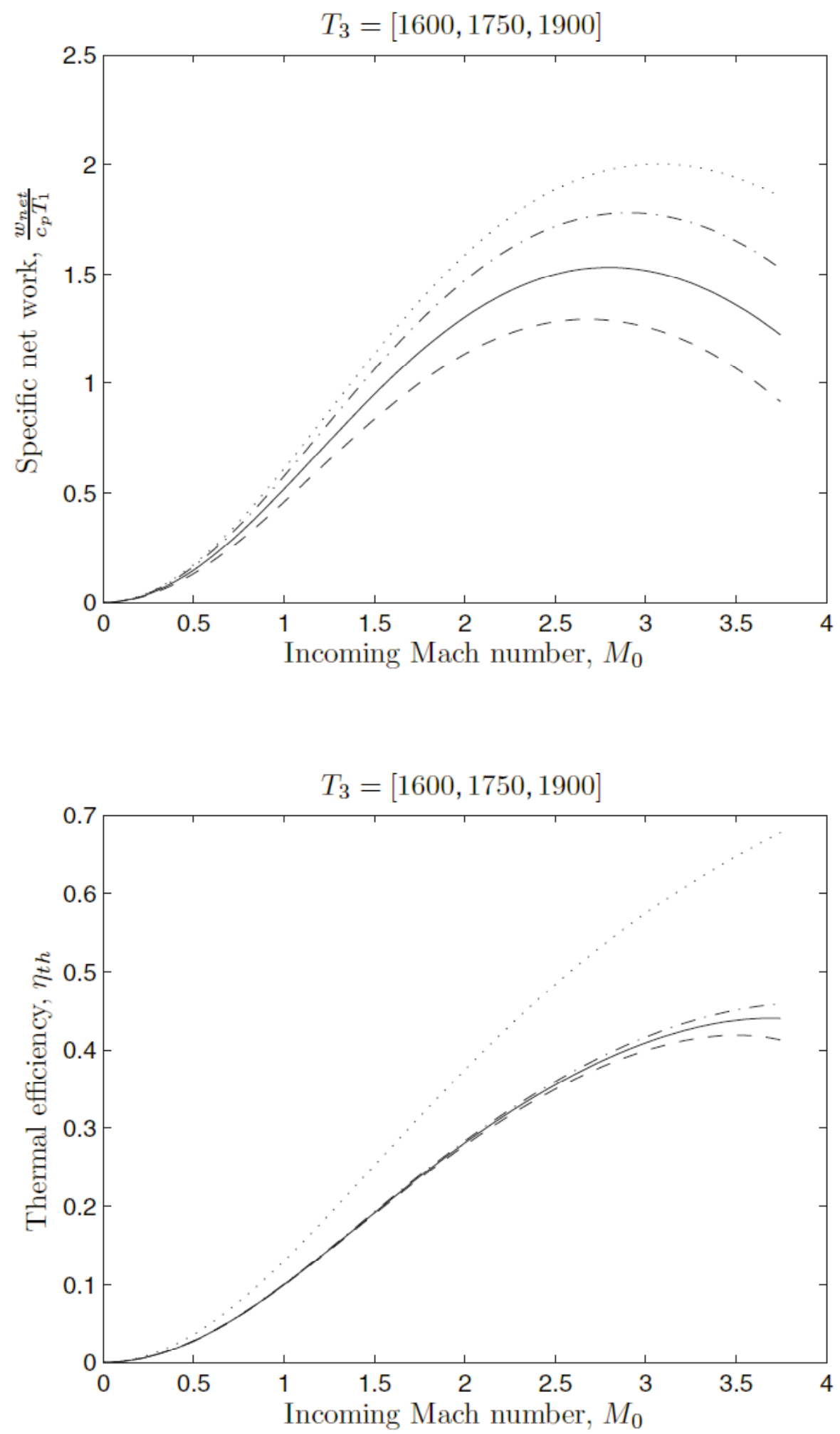

Figure 10. Effect of combustor temperature $T_{3}$ on net work per reactant mass (top) and thermal efficiency (bottom), showing ideal (dotted) and non-ideal system performance with $T_{3}=1600,1750$ and $1900 \mathrm{~K}$ (remaining lines) for baseline values $\eta_{c}=\eta_{b}=\eta_{e}=90 \%$. 
channels may also reduce, or possibly even eliminate, the need for elaborate internal cooling channels or film cooling holes to maintain wall temperatures within maximum material limits. For example, the maximum working temperature for silicon nitride ceramic material is around $1850 \mathrm{~K}$, whereas even with single-crystal turbine blades, thermal barrier coatings, and intricate cooling channels and film cooling the maximum $T_{3}$ currently achieved in modern gas turbine engines is only about $1920 \mathrm{~K}$. This suggests that $T_{3}$ in an inside-out rotary ramjet engine with SiNi ceramic ramjet channels might approach $1850 \mathrm{~K}$ without any cooling, and with only moderate cooling might match the current $1920 \mathrm{~K}$ limit on $T_{3}$ of conventional gas turbines. By implementing comparably elaborate cooling schemes as currently used on gas turbine engines, such an inside-out rotary ramjet system may be able to far exceed the current maximum $T_{3}$ value for conventional turbine engines.

Moreover, in traditional gas turbine-based systems, developing detailed cooling flow paths to maintain leading turbine blade stages within acceptable temperatures under the high tensile stresses to which they are subjected represents a significant part of the overall development cost of an engine. Disruptions of these intricate blade cooling schemes, as may occur in dusty environments, can be a source of turbine failures. Additionally, the internal blade cooling passages and film cooling holes also account for a significant part of the fabrication and maintenance cost of such traditional gas turbine-based systems. By contrast, the inside-out rotary ramjet systems described here eliminate the need for turbine blades and allow for operation with ceramic flow channels that reduce or eliminate the need for internal cooling channels and film cooling holes, and thus have the additional advantage of significantly simplifying the development, production and maintenance of such systems relative to conventional gas turbines.

A further important consideration is the effect of the rotating motion of the ramjet channels in segregating reactants and products within the combustor. As the initially nonrotating reactant mixture enters the ramjet channels and flows along the supersonic diffuser, it passes through a continuous series of infinitesimal waves that compress the flow and thereby cause it to approach the speed of the ramjet channel. In this manner the flow in the channels begins to rotate with the rotor. Upon reaching the end of the compression section, the velocity difference between the flow and the rotating ramjet channel has been reduced to a low subsonic value, and thus the reactant mixture is rotating at nearly the same rate as the rotor. The reactants entering the combustor thus are undergoing a centripetal acceleration nearly equal to $R \Omega^{2}$, where $R$ is the rotor rim radius and $\Omega$ is the rotor rotation rate. Regardless of the rotor radius, the rotor rim speed $U_{C}=R \Omega$ must correspond to an inlet Mach number in the range of about 2 to 3 (see Figs. 6-10) in order for the ramjet channels to operate near their maximum performance. As a result the centripetal acceleration imposed on the flow in the combustor scales as $1 / R$, and thus increases when the rotor radius is reduced. For essentially any reasonable rotor radius, Fig. 11 shows the resulting centripetal acceleration at the rotation rate corresponding to maximum output power. Note that for $R=1 \mathrm{~m}$ the flow is subjected to $90,000 \mathrm{~g}$ 's, while for $R=10$ $\mathrm{cm}$ the flow experiences nearly 1,000,000 g's. Within the combustor, the reactants burn to form low-density combustion products, and this large centripetal acceleration then acts to segregate the low-density products from the high-density reactants. The effects of such rotationally-induced segregation in a combustion system can be striking, even at very low rotation rates, as in the well-known "Emmons fire whirl" [5,6] or "flame tornado" [7]. In a highspeed rotating ramjet, the effects of such centripetally-induced density segregation can be extremely important, since the resulting centrifugal forces acting on the flow in the combustor can be enormous.

In particular, in an "inside-out" rotary ramjet the combustor is located in the radially outermost part of the ramjet flow channel, as indicated in Fig. $5 b$. As a consequence, the centripetal acceleration produces centrifugal forces on reactants and products in the combustor that cause the high-density reactants to be naturally forced radially outward, and thus into the combustor. At the same time, the low-density combustion products produced within the combustor are forced radially inward, and thus out of the combustor. The net effect is to ensure an inflow of reactants into the combustor cavity, and thereby assist with mixing between reactants and products without the need for vortex generators or similar devices. By contrast, in an "outside-out" approach with the combustor located in the radially innermost part of the ramjet channel, the centrifugal forces produce the opposite segregation, causing reactants to move radially outward and the combustor to fill with combustion products. The inside-out approach thus has the major advantage of reducing or eliminating the need for devices to assist with mixing and flameholding in the combustor, which further simplifies the development and fabrication of such rotary ramjet systems.

Lastly, the centripetal accelerations in Fig. 11 show that the advantages of the present inside-out approach to rotary ramjet systems over the previous outside-out approach in $[1,2]$ become increasingly important as the rotary ramjet size is decreased. Even in relatively large-scale rotary ramjet systems with a rotor radius of $O(1 \mathrm{~m})$, as in $[1,2]$, the 90,000 g's centripetal acceleration creates large tensile stresses in the ramjet channel material, and strong segregation of reactants and products in the combustor. In a small-scale system roughly the size of a soda can, with a rotor radius of $O(5 \mathrm{~cm})$, the $1,000,000 \mathrm{~g}$ 's centripetal acceleration makes the conversion of tensile stresses in an 


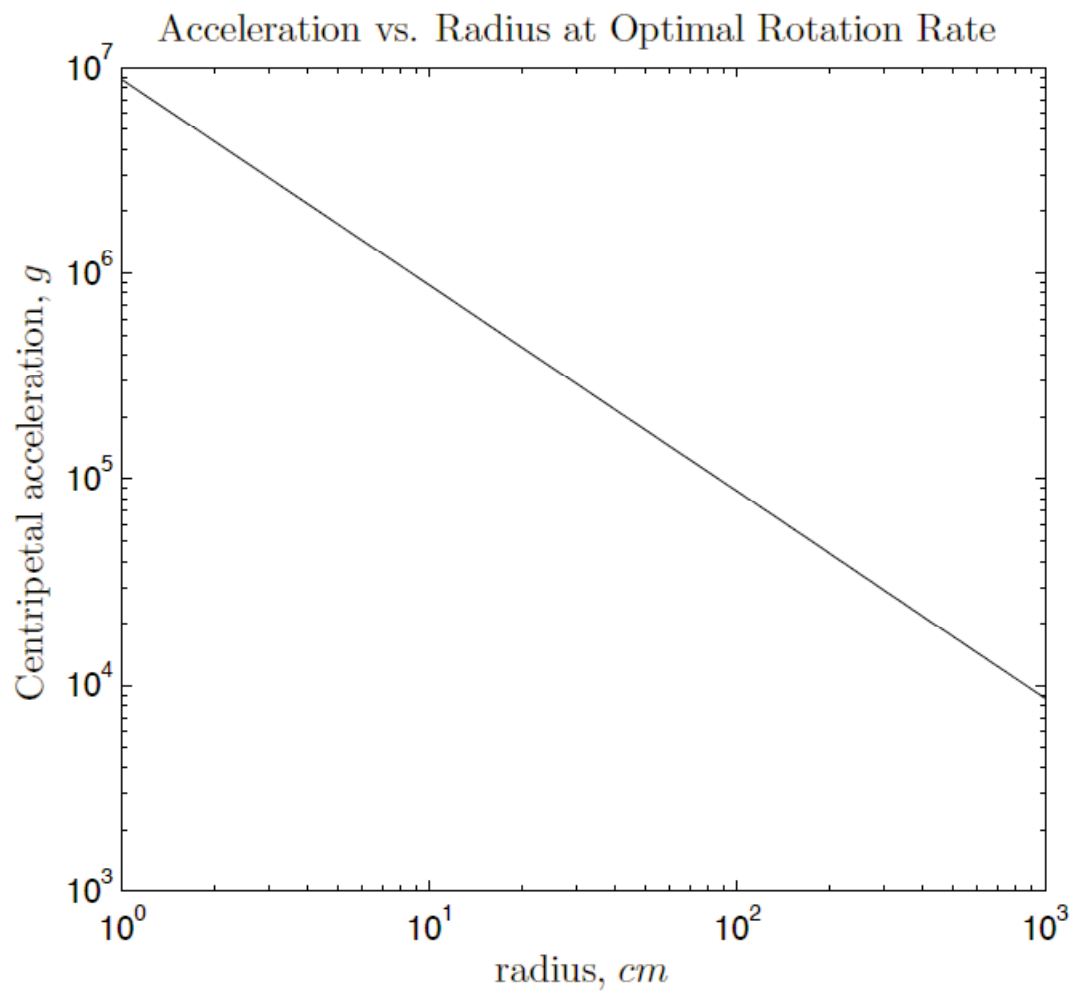

Figure 11. Centripetal acceleration (in $\mathrm{g}$ 's) versus rotor radius (in $\mathrm{cm}$ ) for rotary ramjet system operating at rotation rate corresponding to maximum power for baseline efficiencies $\eta_{c}=\eta_{b}=\eta_{e}=90 \%$ and combustor temperature $T_{3}=1750 \mathrm{~K}$ as in Fig. 6. 
outside-out approach into compressive stresses via an inside-out approach all the more important. Moreover, the even stronger centrifugal segregation of reactants and products in the combustor at this small scale makes the advantageous orientation of the combustor in the inside-out approach essential. While the inside-out approach has important advantages even at the larger scale, these advantages become paramount in smaller-scale systems.

\section{Conclusion}

We have described an improved approach for developing a practical rotary ramjet system for aeropropulsion and power generation applications that eliminates the bladed compressor and turbine found in conventional gas turbinebased turbogenerators, and thereby removes much of the weight, cost, complexity, and endurance limitations of conventional turbine-based systems. Eliminating the rotating bladed turbine also allows the combustor in a ramjet system to be operated at higher overall combustion temperatures, and thereby provides higher efficiencies than conventional bladed turbine engines can achieve at the same compression ratio.

In the present approach these components are replaced with an "inside-out" ceramic ramjet rotor that rotates around a simple concentric ceramic stator at supersonic speeds to allow an efficient Brayton cycle within varyingarea ramjet flowpaths formed in the ceramic rotor rim. The inside-out design places the ramjet flowpath on the inside rim of the rotor, where it is under purely compressive centrifugal loading. This allows high-temperature ceramic materials to be used through the ramjet channels. Such an all-ceramic flowpath in the inside-out ramjet flow channels may reduce, or possibly even eliminate, the need for elaborate internal cooling channels or film cooling holes to maintain wall temperatures within maximum material limits, and thus has the additional advantage of significantly simplifying the development, production and maintenance of such systems.

The all-ceramic ramjet flowpath made possible by the inside-out approach allows substantially higher combustor exit temperatures $T_{3}$ than are possible with a conventional bladed turbine or with the metal-based "outside-out" ramjet rotor in $[1,2] . T_{3}$ in an inside-out rotary ramjet engine with $\mathrm{SiNi}$ ceramic ramjet channels may approach $1850 \mathrm{~K}$ without any cooling, and with only moderate cooling may match the current $1920 \mathrm{~K}$ limit on $T_{3}$ of conventional gas turbines. By implementing comparably elaborate cooling schemes as currently used on gas turbine engines, such a rotary ramjet system may substantially exceed the current $T_{3}$ limit for conventional turbine engines. The increased combustor temperature provides a substantial increase in maximum power and increased thermal efficiency when operating at maximum power. The ability to use high-temperature ceramic materials throughout the ramjet channel provides both a direct increase in maximum power due to the $T_{3}$ effect on $w_{\text {net }}$, and a secondary increase in thermal efficiency by avoiding gradual loss of wall material in the nozzle throat to maintain the optimal $A_{e} / A^{*}$ and thereby obtain an added $\eta_{e}$ benefit on $\eta_{t h}$.

Furthermore, in this inside-out rotary ramjet approach the centrifugal forces acting on reactants and products in the combustor cause the high-density reactants to be forced radially outward, and thus into the combustor. At the same time the low-density combustion products formed in the combustor are forced radially inward, and thus out of the combustor. The net effect is to ensure an inflow of reactants into the combustor cavity, and thereby assist with mixing between reactants and products without the need for vortex generators or similar devices. Reducing or eliminating the need for such additional mixing and flameholding devices in the combustor further simplifies the development and fabrication of practical rotary ramjet systems, and may overcome one of the key impediments in previous attempts at developing rotary ramjet systems $[1,2]$.

Finally, while the inside-out approach to rotary ramjet systems has important advantages even in large-scale systems, these advantages become increasingly important as the device size is reduced. The $1 / R$ increase in centripetal acceleration with rotor radius $R$ makes the conversion of the tensile stresses that occur in an outside-out approach into compressive stresses via the inside-out approach all the more important as the device size becomes smaller. Moreover, the stronger centrifugal segregation of reactants and products in the combustor with decreasing device size makes the advantageous orientation of the combustor in the inside-out approach essential.

\section{References}

${ }^{1}$ Lawlor, S.P. (2001) Ramjet engine for power generation. U.S. Patent No. 6,298,653. U.S. Patent and Trademark Office, Washington, D.C.

${ }^{2}$ Steele, R.J. et al. (2001) Development and testing of a pre-prototype Mach 2 Ramgen engine. Final Report, DOE Award No. DE-FC26-00NT40915, Department of Energy, Washington, D.C.

${ }^{3}$ Builder, C.H. (1964) On the thermodynamic spectrum of airbreathing propulsion. AIAA paper 64-243, AIAA, Washington, D.C. 
${ }^{4}$ Hill, G.H. and Peterson, C.R. (1992) Mechanics and Thermodynamics of Propulsion. Addison-Wesley Publishing Co., New York.

${ }^{5}$ Emmons, H.W. and Ying, S.-J. (1967) Proceedings of the $11^{\text {th }}$ (International) Symposium on Combustion. p. 475, The Combustion Institute, Pittsburgh, PA.

${ }^{6}$ Beer, J.M., Chigier, N.A., Davies, W. and Bassindale, K. (1971) Laminarization of turbulent flames in rotating environments. Combustion and Flame, Vol. 16, No. 1, pp. 39-45, The Combustion Institute, Pittsburgh, PA.

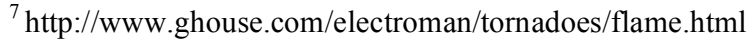

